\title{
Material balance of corrosion inhibitors. Distribution at lab testing and field application
}

\author{
I. S. Sivokon \\ OJSC Transenergostroi, Derbenevskaya nab. 7, bldg. 10, Moscow, \\ 115114 Russian Federation.E-mail: issiv64@gmail.com
}

\begin{abstract}
This article may produce an impression that it deals with low priority issues of pipelines corrosion inhibitor protection processes and corrosion inhibitors (CI) lab testing techniques. However, it is proved that clear understanding of CI active molecules location makes it possible to identify the causes of CI inefficiency and can be used to produce recommendations to adjust lab testing and $\mathrm{CI}$ efficiency evaluation techniques.

Inadequate attention paid to the CI distribution material balance may result in:

- Wrong choice of CIs at lab tests

- Excessive or insufficient concentration selection to ensure required protective action at field applications
\end{abstract}

A series of recommendations is proposed by the author based on a discussion and review of the cases described in the article. Research guidelines are proposed as knowledge of CI properties is essential to overcome current shortcomings.

Key words: corrosion inhibitors, testing techniques, oilfield pipelines.

Received: February 26, 2013.

doi: $10.17675 / 2305-6894-2013-2-2-102-114$

\section{Corrosion inhibitors material balance used for efficient field application and lab tests}

Inhibition corrosion control, similar to other technologies based on production chemicals application, is widely spread in the industry, though it can't be considered a well understood process. A seemingly simple procedure - inject a right amount of a correctly selected inhibitor into a right location and required results will be achieved - leads to neglecting numerous specific features of the inhibition protection process.

Essentially, the process is about adding a special compound in vanishingly small concentrations of 5 to $15 \mathrm{ppm}$ to some liquid transported by pipelines. As a result, a protective film composed of several molecular layers is produced on the internal metal surface reducing the corrosion rate by 10 and more times.

Due to pure economics, a large-scale application of CIs requires a minimum amount to be added for treatment while still ensuring a control action, still avoiding any redundancy. Hence the need to understand what for, except forming the protection film, the CI active compound can be utilized. 
The fundamental material balance equations based on practice and common sense make it possible to identify risks that occur while inhibiting; it is also an opportunity to develop extremely valuable recommendations designed to enhance inhibitor protection efficiency.

A similar material balance-based approach is appropriate for CI laboratory testing as well, since lab test results are used to develop and select most efficient CIs for industrialscale testing to be further rolled-out into large-scale field application.

\section{CI material balance field application. Continuous treatment}

Let us consider a most common practical situation when CIs are used to control pipeline internal corrosion by a continuous treatment technique. The technique includes maintaining a pre-set CI concentration designed to assure a required protection effect of usually at least $90 \%$. Since the majority of the CIs form a protective film on the surface, it is logical to assume that the CI concentration is to provide a balance between the rate of film forming and the rate of its destruction. The CI protective film degradation is determined not only by CI properties, but by flow hydraulics, solids content and other factors as well.

So, a stationary process is realized during the continuous treatment when the amount of $\mathrm{CI}$ fed into pipelines is equal to the amount of CI leaving the pipelines along with transported fluids.

$-\mathrm{CI}$ in the pipelines ${ }^{1}$ used to transport high water cut wells production may be found in:

- oil, $M_{\mathrm{o}}$

- water, $M_{\mathrm{w}}$;

- oil-in-water emulsion and water-in-oil emulsion in oil on the interfacial surface, $M_{\mathrm{e}}$;

- on solids, including salt crystals that set down and produce scaling due to saturation of water by calcium and barium ions, $M_{\mathrm{s}}$;

Generally speaking, CIs may be found in the gas phase as well; however, this factor is significant only for a very limited group of CIs used to inhibit gas pipelines and is not considered in this paper.

Below is the material balance equation:

$$
M=M_{\mathrm{w}}+M_{\mathrm{o}}+M_{\mathrm{e}}+M_{\mathrm{s}},
$$

where $M$ is the weight of CI arriving to the pipeline as it is continuously treated.

Besides, CI is found on the pipeline surface as the protective film; its weight $M_{\mathrm{f}}$ is calculated using the equation:

\footnotetext{
${ }^{1}$ In this case oil gathering lines only are considered - pipelines that are used within a field to collect crude oil produced by wells. Other types of oil field pipelines, for example, oil pipelines that transport treated or commercial oil, or water pipelines that belong to reservoir pressure maintenance systems are insignificant applications from the material balance point of view since water or oil content in these cases may be too small (0.001), sometimes exceeding 2-3 per cent.
} 


$$
M_{\mathrm{f}}=S \cdot \rho,
$$

where $S$ is the area of the pipeline internal surface and $\rho$ is the density of the protective film in $\mathrm{g} / \mathrm{m}^{2}$.

In order to make the equation (1) practical, the corresponding weight values of $\mathrm{CI}$ in water, emulsion and on solids shall be converted into practically measured values, for example:

$$
M_{\mathrm{s}}=C_{\mathrm{s}} \cdot V \cdot \delta,
$$

where $C_{\mathrm{s}}$ is the concentration on the solids, $\mathrm{mg} / \mathrm{l} ; V$ is the volume of the pipeline in liters; $\delta$ is the dimensionless factor - CI consumption per unit of weight of the solids. The value of $\delta$ depends on the size and surface properties of solids as well as on the CI properties.

Paper [3] includes the results of measuring the value of $\delta$ for CI Sonkor 9011 and ETK 5722 on 4 types of solids: shale, calcium carbonate, sand and iron sulfide. It was established that the tested CIs adsorb mostly on shale and iron sulfide, while the value of $\delta$ ranges from $0.01 \mathrm{mg} / \mathrm{mg}$ for sand and up to $1.1 \mathrm{mg} / \mathrm{mg}$ for iron sulfide. It is also established that the value of $\delta$ increases along with the concentration growth: as the CI concentration in water increases 10 times, $\delta$ grows by 5 to 10 times. A calculation of the effective concentration tested for a real oil gathering pipeline with a usual concentration of solids exceeding $100 \mathrm{mg} / 1$ using $25 \mathrm{mg} / 1 \mathrm{CI}$ concentration, the real concentration will amount to $15 \mathrm{mg} / \mathrm{l}$ maximum. In other words, CI losses for solids adsorption are as high as at least $40 \%$.

The value of $M_{\mathrm{e}}$ may be computed provided the emulsion volume and specifications are known, for example, the average size of oil and water drops and their quantity per unit of volume. This data may be used then to compute the area of the adsorbing CI interphase boundary. Then:

$$
M_{\mathrm{e}}=V_{\mathrm{e}} \cdot N_{\mathrm{wd}} \cdot \pi d^{2} \cdot \rho_{\mathrm{o}-\mathrm{w}},
$$

where $V_{\mathrm{e}}$ is the volume of emulsion, $\mathrm{m}^{3} ; N_{\mathrm{wd}}$ is the number of oil or water drops in a unit of the emulsion volume, $\mathrm{m}^{-3} ; \rho_{\mathrm{o}-\mathrm{w}}$ is the CI density at the interphase boundary between oil and water, $\mathrm{g} / \mathrm{m}^{2}$.

$M_{\mathrm{w}}$ and $M_{\mathrm{o}}$ may be calculated provided the CI concentration in water or oil is known. Numerous techniques are available in traditionally equipped chemical laboratories for determining CI concentration in water. Besides, most CIs feature solvability or dispersability both in water and oil. Such CIs can be analyzed by using distribution coefficient $\zeta$ as one of their characteristics that can be measured under laboratory conditions and calculated using the equation below:

$$
\zeta=C_{\mathrm{o}} / C_{\mathrm{w}}
$$

where $C_{\mathrm{o}}$ is the CI equilibrium concentration in oil and $C_{\mathrm{w}}$ is the CI equilibrium concentration in the two-phase system made up of oil and water with CI added in doses close to those of industrial applications. 
If $\zeta$ and $C_{\mathrm{w}}$ are known, it is easy to calculate $C_{\mathrm{o}}$ and hence to determine $M_{\mathrm{o}}$ and $M_{\mathrm{w}}$.

$$
\begin{gathered}
C_{\mathrm{z}}=\left(C_{\mathrm{w}} \cdot \eta+C_{\mathrm{o}} \cdot(1-\eta)\right), \text { consequently } \\
C_{\mathrm{w}}=\left(C_{\mathrm{z}}-C_{\mathrm{o}} \cdot(1-\eta)\right) / \eta \text { and } \\
C_{\mathrm{o}}=\left(C_{\mathrm{z}}-C_{\mathrm{w}} \cdot \eta\right) /(1-\eta),
\end{gathered}
$$

where $\eta$ is the share of water in the pipeline-transported fluid, while $C_{\mathrm{z}}$ is the concentration of the inhibitor delivered to the pipeline during the continuous treatment and calculated proceeding from the specific liquid (oil + water).

On the other hand, if $C_{\mathrm{z}}$ is known (this is a known value since it is obtained as CI is delivered), $C_{\mathrm{o}}$ and $C_{\mathrm{w}}$ can be calculated as the CI property making use of distribution coefficient $\zeta$

$$
\begin{gathered}
C_{\mathrm{z}}=C_{\mathrm{w}} \cdot \eta+\mathrm{C}_{\mathrm{w}} \cdot \zeta \cdot(1-\eta), \text { consequently } \\
C_{\mathrm{w}}=C_{\mathrm{z}} /(\eta+\zeta \cdot(1-\eta))
\end{gathered}
$$

Since the internal corrosion of low carbon steel pipelines takes place because of the contact between the tubular inner surface and salt water, CI concentration in water environment $C_{\mathrm{w}}$ is more significant for producing the CI protective film on the surface compared with the $C_{\mathrm{o}}$ - the CI concentration in oil, especially when the fluid flow in the pipeline is laminated and there are certain areas on the pipeline inner surface that do no come into contact with the oil phase. Figure 1 shows the calculated values of $C_{\mathrm{w}}$ for CI featuring different values of $\zeta$ and water content $\%$ in transported oil.

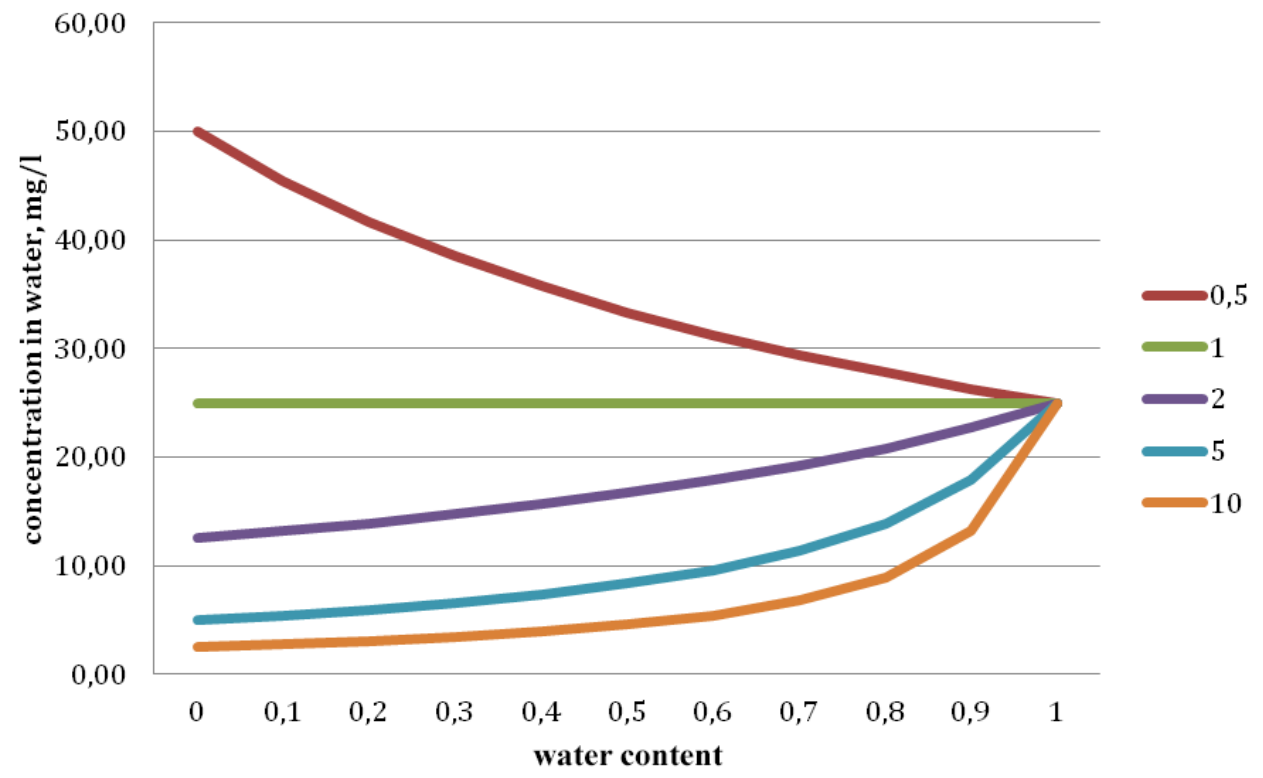

Fig. 1. Calculated CI concentration in the water phase as it is transported by the oil gathering line under $25 \mathrm{ml} / 1$ continuous treatment at $0.5,1,2,5$ and 10 distribution coefficients and water content ranging from 0 to 1 . 
In practice, most common corrosion inhibitors applied to protect pipelines from internal corrosion [1] are soluble and can be easily dispersed in water, with $\zeta$ ranging from 1 to 10 or more. The graph demonstrates that such CIs are more effective when a higher water cut fluid of over $80 \%$ is transported, since $C_{\mathrm{w}}$ is almost equal to $C_{\mathrm{z}}$ delivered to the pipeline. To protect pipes that transport lower water cut fluids as laminated flows, CIs insoluble in oil are required, since such inhibitors are capable of creating concentrations in the water phase sufficient to maintain the protective film at continuous treatment. As for $\zeta$ for these products, it should be equal to 2 maximum.

It has to be noted that for CIs that are multi-component products, the values of $\zeta, C_{\mathrm{o}}$ and $C_{\mathrm{w}}$ can't be reliably defined and, consequently measured with a sufficient precision. These may be used only for CI applicability assessment and risk evaluation at industrial applications.

Let's return to equation (1). Listed below are the factors reducing the CI concentration in the water and oil phase:

$-\zeta$ - the CI distribution coefficient reflecting its ability to be present in the water phase, ref. equations $(5,6.1,6.2,6.3,7)$;

$-\delta$, which defines the CI ability to be deposited on solids, ref. equation (3);

$-C_{\mathrm{s}}$ - content of solids in transported fluid;

- transported emulsion properties: $V_{\mathrm{e}}$ - volume of emulsion or its share in the transported fluid volume; $N_{\mathrm{wd}}$ - quantity of oil or water drops in a unit of the emulsion volume, drops per $\mathrm{m}^{3} ; d$-average drop diameter in emulsion, $\mathrm{m} ; \rho_{\mathrm{o}-\mathrm{w}}-\mathrm{CI}$ density at the oil/water interphase boundary, $\mathrm{g} / \mathrm{m}^{2}$; ref. equation (4);

$-\eta-$ the share of water in pipeline transported liquid.

The above-listed factors can be measured with various precision in labs and calculated on the basis of available process parameters recorded at pipelines where CIs are used to protect from internal corrosion.

The present-day high level of oil production and transportation automation makes it possible to calculate the pipeline process parameters using current data updated on a daily basis or even more frequently. Three sets of initial data are required to properly manage continuously treated pipelines:

1. CI process characteristics required for calculation and monitoring of the CI material balance in the pipeline;

2. Protective action properties: the CI concentration required to maintain the protective film ensuring at least $90 \%$ corrosion rate reduction depending on the flow velocity, temperature, oxygen content and solids impact;

3. Operational hydraulics of protected pipelines and transported fluid properties at homogenous sections, including solids concentration, emulsion properties and volume, and, provided a significant impact on the CI protective properties is known, the chemical composition of water and oil. 
The first two sets of initial data are constant in time and are dependent on CI and its quality stability, while data of the third set tend to alter along with activities at the producing wells. Thus, as day-to-day changes occur, adjustments are to be made in the CI concentrations as well. This is an opportunity to proactively change the treatment process as opposed to the current practice of reactive changes with a delay by one or two months since the response is based on a limited scope of corrosion monitoring and/or findings of accident and incident investigations of leaks caused by internal corrosion.

At present $\mathrm{CI}$ application practice is based on using a single indicator - concentration (mg/l) that was demonstrated by the CI as it was tested at a certain section of the protected pipeline to prove the protection efficiency of at least $90 \%$.

\section{CI material balance at industrial application. Protective film formation}

The CI material balance described by equation (1) under steady-state conditions fails to depict the CI protective film formation on the pipeline internal surface. Meanwhile, the protective film formation is a key stage in ensuring efficient inhibitor protection and requires significant costs. Consequently, this question along with determining the CI regular concentration calls for comprehensive engineering efforts based on the pipeline system and CI physical properties.

Equation (2) includes the weight of CI deposited on the inner surface of the pipeline as a protective film. Moreover, as demonstrated by laboratory experiments, a certain time is required for the film to be produced which depends on numerous factors, e.g., hydraulic conditions, inner surface roughness, temperature and CI concentration in the transported fluid.

The residual content of $\mathrm{CI}$ is used as the indicator of the protective film formation process. Figure 2 shows a typical chart of CI concentration in water required for protective film formation during batch treatment. Sampling at such experiments takes place at the pipeline final point and the CI concentration stabilization serves as the indicator to prove the completion of the film formation process or, in other words, the system made up of the pipeline, liquid and CI has transferred into its saturated condition and the incoming CI volume is equal to the outgoing $\mathrm{CI}$ at the end of the pipeline.

It has to be noted that the technique used in this case for determining the duration of the CI batch treatment is not precise since inaccuracies are high as CI concentration in water is measured and also because the rate of protective film formation depends on a number of factors. So, chances are high that the process is completed only at certain sections of the pipeline, while whether film was formed along the whole pipeline is not known because of the low accuracy of measuring CI concentration and possible short distances of the no-film sections. So, measurement results may provide a constant value within the specified tolerance range. 


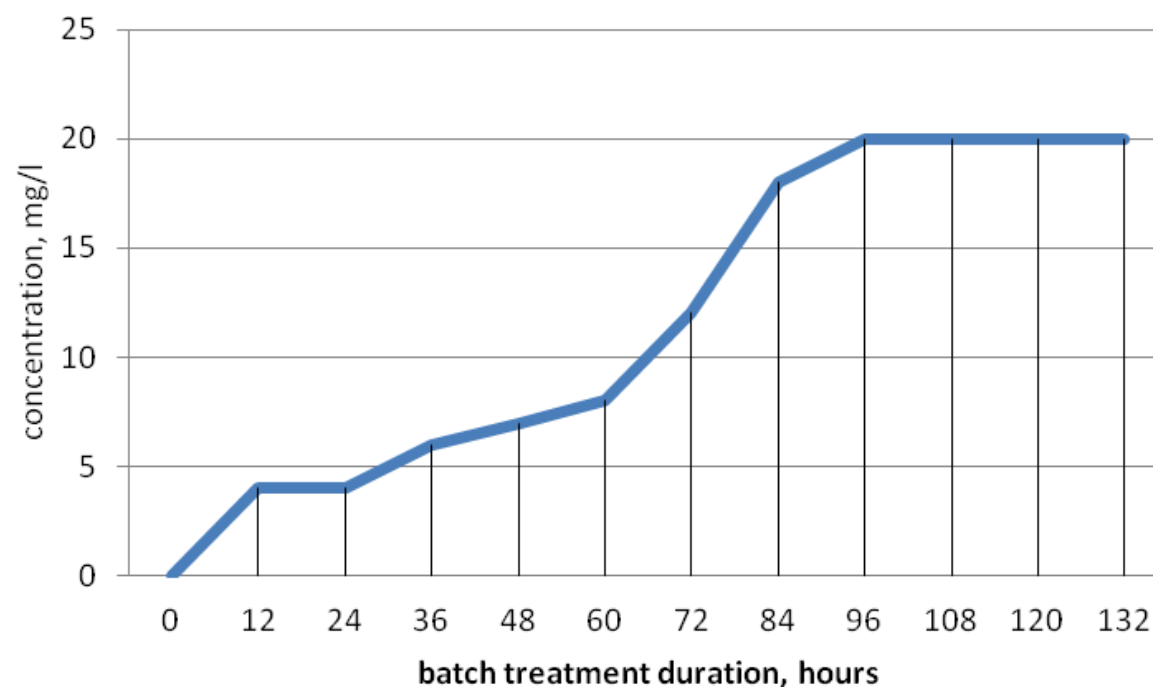

Fig. 2. CI concentration $(\mathrm{mg} / \mathrm{l})$ dynamics at pipeline exit point as protective film is formed during batch treatment

The following proposals may be used as practical recommendations for CI batch treatment of pipeline systems:

1. The volume of CI used for batch treatment and its duration shall be selected in multiples compared to the CI weight in the protective film, ref. equation (2) and time required for its formation;

2. The multiplying factor at batch treatment shall be at least 2 or 3 when applied to:

a. Concentration: it should exceed the estimate by 2-3 times,

b. Weight delivered during the CI batch treatment: at least 2-3 times higher compared to the calculated value obtained by equation (2),

c. Time required for formation of the protective film or duration of the batch treatment shall be at least 2-3 times longer compared to the CI film formation duration determined under laboratory conditions;

3. The CI concentration stabilization at the exit of the treated pipeline shall be used as the batch treatment sufficiency indicator.

A batch treatment was tested for 2 weeks within a major project designed to implement inhibition in TNK-Nyagan in 2004 [2]. No pipelines have been treated in this company before. No accidents or incidents were recorded within less than two months after the treatment at previously leaking pipelines of the oil gathering system of Talinskoe oil and gas field. So, there all reasons to state that cost saving is not a good idea at the batch treatment stage of inhibition technology.

\section{CI material balance at laboratory testing}

The conditions for CI lab testing significantly differ from any field application environment. The reason for this difference is that in all types of lab experiments, systems subjected to corrosion protection and film formation processes are closed, i.e. there is no 
delivery of additional substances to the system during the test except, for example, carbon dioxide during bubble tests.

$\mathrm{CI}$ lab testing is characterized by a significant duration required to achieve the equilibrium state when the corrosion rate, protection film formation and other processes are properly balanced. The CI material balance for any lab testing type excluding testing of flowing (open) systems can be describe using the following equation:

$$
M=M_{\mathrm{w}}+M_{\mathrm{h}}+M_{\mathrm{ms}}+M_{\mathrm{a}},
$$

where $M$ is the weight of CI delivered to the cell; $M_{\mathrm{w}}$ is the weight of $\mathrm{CI}$ in the test water solution; $M_{\mathrm{h}}$ is the weight of CI in test hydrocarbon phase (if present in the test), $M_{\mathrm{ms}}$ is the weight of CI used for protection film formation on metal samples located into the cell and used for corrosion rate evaluation; $M_{\mathrm{a}}$ is the weight of CI adsorbed at the cell, electrodes, mixer and other surfaces in contact with the model corrosion medium.

Moreover, the material balance equation may include CI present in the emulsion, if applicable, and CI adsorbed on solids and particulate matters of salt crystals in case testing is performed in an environment subjected to salt and corrosion products deposition or when solids impact on the CI protective capability is investigated. The additional elements of the material balance are not applicable in most CI lab tests, so they are not considered hereafter.

In order to further simplify the analysis, the equation (8) may be converted into:

$$
C_{\mathrm{z}} \cdot V=C_{\mathrm{w}} \cdot V \cdot \eta+C_{\mathrm{w}} \cdot \zeta \cdot V \cdot(1-\eta)+S_{\mathrm{ms}} \cdot \rho_{\mathrm{ms}}+S_{\mathrm{a}} \cdot \rho_{\mathrm{a}},
$$

where $\eta$ is the fraction of water in test liquid; $S_{\mathrm{ms}}$ and $S_{\mathrm{a}}$ are the area of samples and equipment cell, $\mathrm{cm}^{2} ; \rho_{\mathrm{ms}}$ and $\rho_{\mathrm{a}}$ are the density of film on samples and walls and cell equipment, $\mathrm{mg} / \mathrm{cm}^{2}$.

The material balance equation provided above makes it possible to make an immediate conclusion: CI that are non-soluble and poorly dispersed in the test water solution it is not adequate to perform comparison tests without accounting for the hydrocarbon medium and its contact with samples and electrodes being researched. Such tests are not feasible since it is not known how much CI is required for the protection film formation and what CI quantities will be simply absorbed on the cell walls and equipment. Besides, no conditions exist for the protection film formation during the test since the test medium does not contain CI. In this case the presence of the hydrocarbon phase as the CI carrier that ensures manageable and repeatable test-to-test CI logistics needed for the protection film formation is required. The following material balance equation may be used in such cases:

$$
C_{\mathrm{z}} \cdot V=C_{\mathrm{h}} \cdot V \cdot(1-\eta)+S_{\mathrm{ms}} \cdot \rho_{\mathrm{ms}}+S_{\mathrm{a}} \cdot \rho_{\mathrm{a}}
$$

where $C_{\mathrm{h}}$ is the CI concentration in the test hydrocarbon phase, assuming CI in the water phase is practically absent.

Now, let's get back to the material balance equation (9) that describes most tests and is applicable in case of water-soluble and water-dispersible CIs to demonstrate a potential impact of the cell cup size and cup shape on the test results. The CI bubble test conducted in accordance with the procedure [4] will serve as an example. Usually, in such tests the 
remaining concentration of CIs that are well-dispersed in water upon completion of the test is $10-15 \mathrm{mg} / \mathrm{l}$ with the concentration of $20 \mathrm{mg} / \mathrm{l}$. Let's consider two fully equal tests in test water medium but conducted using different form cells, ref. Table 1.

Table 1. Physical dimensions of test cells and CI concentration. $20 \mathrm{mg} / \mathrm{l} \mathrm{CI}$ concentration.

\begin{tabular}{|c|c|c|c|c|}
\hline \multirow[b]{2}{*}{$\begin{array}{c}\text { Cells physical properties and CI concentration } \\
\text { measurement results }\end{array}$} & \multicolumn{2}{|c|}{ Cell 1} & \multicolumn{2}{|c|}{ Cell 2} \\
\hline & $\begin{array}{c}\text { Water test } \\
\text { medium } \\
\text { testing }\end{array}$ & $\begin{array}{l}\text { Two-phase } \\
\text { medium, } \\
10 \% \text { of oil }\end{array}$ & $\begin{array}{l}\text { Water test } \\
\text { medium }\end{array}$ & $\begin{array}{c}\text { Two-phase } \\
\text { medium, } \\
10 \% \text { of oil }\end{array}$ \\
\hline Diameter, cm & \multicolumn{2}{|c|}{10} & \multicolumn{2}{|c|}{7} \\
\hline Height, cm & \multicolumn{2}{|c|}{10} & \multicolumn{2}{|c|}{17} \\
\hline$S_{\mathrm{a}}-$ cell surface area, $\mathrm{cm}^{2}$ & \multicolumn{2}{|c|}{314} & \multicolumn{2}{|c|}{374} \\
\hline$V$ - Cell volume, liter & \multicolumn{2}{|c|}{0.785} & \multicolumn{2}{|c|}{0.654} \\
\hline$C_{\mathrm{z}}-\mathrm{CI}$ treatment concentration, $\mathrm{mg} / \mathrm{l}$ & \multicolumn{2}{|c|}{20} & \multicolumn{2}{|c|}{20} \\
\hline$C_{\mathrm{w}}-\mathrm{CI}$ final concentration in water, $\mathrm{mg} / \mathrm{l}$ & 15 & 10 & 12.85 & 7.69 \\
\hline$\zeta-\mathrm{CI}$ distribution coefficient & $\mathrm{n} / \mathrm{a}$ & 3 & $\mathrm{n} / \mathrm{a}$ & 3 \\
\hline$S_{\mathrm{ms}}-$ samples area, $\mathrm{cm}^{2}$ & \multicolumn{4}{|c|}{6} \\
\hline$\eta-$ Water share in test medium & 1 & 0.9 & 1 & 0.9 \\
\hline
\end{tabular}

Equation (9) for cell 1 is:

$$
20 \cdot 0.785=15 \cdot 0.785+314 \cdot \rho_{\mathrm{a}}+6 \cdot \rho_{\mathrm{ms}}
$$

- one-phase test medium

$$
20 \cdot 0.785=10 \cdot 0.785+10 \cdot 3 \cdot 0.785 \cdot(1-0.9)+314 \cdot \rho_{\mathrm{a}}+6 \cdot \rho_{\mathrm{ms}}
$$

- two-phase test medium.

Let's also write down the equation for cell 2 . The CI residual concentration at the end of the test is not known, but it is possible to assume that $\rho_{\mathrm{a}}$ and $\rho_{\mathrm{ms}}$ values for both tests are equal since these values do not depend on the cell form:

$$
\begin{gathered}
20 \cdot 0.654=C_{\mathrm{x} 1} \cdot 0.654+374 \cdot \rho_{\mathrm{a}}+6 \cdot \rho_{\mathrm{ms}} \\
20 \cdot 0.654=C_{\mathrm{x} 2} \cdot 0.654+C_{\mathrm{x} 2} \cdot 3 \cdot 0.654 \cdot(1-0.9)+374 \cdot \rho_{\mathrm{a}}+6 \cdot \rho_{\mathrm{ms}}
\end{gathered}
$$

CI concentrations $C_{\mathrm{x} 1}$ and $C_{\mathrm{x} 2}$ for the second test can be computed by subtracting equations for the one-phase (11.1 and 11.3) and two-phase (11.2 and 11.4) media, respectively, and the results are as follows:

$$
\begin{gathered}
2.62=11.775-C_{\mathrm{x} 1} \cdot 0.654-60 \cdot \rho_{\mathrm{a}} \\
2.62=7.850-C_{\mathrm{x} 2} \cdot 0.654+2.355-0.196 \cdot C_{\mathrm{x} 2}-60 \cdot \rho_{\mathrm{a}}
\end{gathered}
$$


Since the samples area is approximately 100 times smaller than the area of the cells walls and equipment, the weight of CI used for the protection film formation on the samples may be neglected. Therefore, $\rho_{\mathrm{a}}$ value may be calculated based on the equations (11.1) and (11.3): $\rho_{\mathrm{a}}=0.0125 \mathrm{mg} / \mathrm{cm}^{2}$ based on the equation (11.1) for the one-phase test medium testing and $\rho_{\mathrm{a}}=0.0175 \mathrm{mg} / \mathrm{cm}^{2}$ based on the equation (11.2) for the two-phase test medium testing in cell 1 .

With $\rho_{\mathrm{a}}$ value known for each set of tests, values of $C_{\mathrm{x} 1}$ and $C_{\mathrm{x} 2}$ may be calculated using the equations (12.1) and (12.2):

$$
\begin{gathered}
C_{\mathrm{x} 1}=(11.775-2.62-0.75) / 0.654=12.85 \\
C_{\mathrm{x} 2}=(7.850-2.62+2.355-1.05) /(0.654+0.196)=7.69
\end{gathered}
$$

Let us review another scenario for cell 1 during the test of the one-phase solution but for a different CI with, for example, cell walls film density two times higher $\left(0.0250 \mathrm{mg} / \mathrm{cm}^{2}\right.$ instead of $0.0125 \mathrm{mg} / \mathrm{cm}^{2}$ ). The results of the same calculation shows that at the end of the test the CI residual concentration in water will be just $10 \mathrm{mg} / \mathrm{l}$, while the same concentration is $15 \mathrm{mg} / \mathrm{l}$ for CI with lower deposits on the cell walls.

Paper [3] contains measurements of CI concentration reduction due to lab cell walls adsorption. It was established that corrosion inhibitor Sonkor 9011 CI practically does not produce deposits on the cell glass walls, while ETK $5277 \mathrm{CI}$ when delivered at $25 \mathrm{mg} / \mathrm{l}$ in $5 \mathrm{~h}$ was present in the water phase at the concentration of $21.6 \mathrm{mg} / \mathrm{l}$.

The examples of the material balance impact during CI lab testing provided above show that protective action measurement results for equal amounts of various CIs may not be used for comparison analysis. The reason behind this is that the true concentration resulting in formation of the CI protection film during lab testing is highly dependent on the cells geometry and surface properties as well as on the properties of the CI itself.

The impact of the cells geometrical dimensions and forms as well as samples and electrodes preparation on the CI testing result may be demonstrated using protection effect testing conducted at the oil fields by the Institute of Energy Resources Transportation Problems (IPTER) and British engineering company CAPSIS [6]. CI lab testing activities were performed by the above mentioned organizations at the same time and independently using the same CI samples taken at oil fields, identical reservoir water test solution and established technique for conducting bubble tests for carbon dioxide (sweet) and combined corrosion with addition of hydrogen sulfide (sweet and sour). The differences were in the cells form, hydrocarbon phase, samples/electrodes and auxiliary instruments for corrosion rate measurement using the polarization resistance measuring technique. The comparison results of the measurements conducted during the bubble tests are shown in Table 2.

The Samotlor field reservoir water was used as a test solution during the carbon dioxide corrosion tests:

\begin{tabular}{ccccc}
\hline Component & $\mathbf{N a C l}$ & $\mathbf{C a C l}_{\mathbf{2}}$ & $\mathbf{M g C l}_{\mathbf{2}}$ & $\mathbf{N a H C O}_{\mathbf{3}}$ \\
\hline Quantity, g/l & 11.2 & 1.629 & 0.328 & 0.453 \\
\hline
\end{tabular}


Paper [5] deals with the hydrocarbon phase represented by oil of the same field that accounts for $20 \%$ of the test solution volume. The tests were conducted with $\mathrm{CO}_{2}$ partial pressure of $0.1 \mathrm{~atm}$. The initial corrosion rate is $1.35 \mathrm{~mm} /$ year. The paper [6] deals with the hydrocarbon phase represented by 1:1:1 kerosene, toluene and benzene mixture with $\mathrm{CO}_{2}$ partial pressure of $1 \mathrm{~atm}$. and the initial corrosion rate of $2.5-3.5 \mathrm{~mm} / \mathrm{year}$.

The CI efficiency comparison based on the results provided in Table 2 allows to make an unambiguous conclusion of Sonkor 9011 as the most efficient CI based on the data obtained by the Institute of Energy Resources Transportation Problems. However, no similar conclusion can be made based on the CI efficiency measurements performed by CAPSIS, since Sonkor 9011 and Dodicor 4712 CI are comparable for the concentration of $20 \mathrm{mg} / \mathrm{l}$, while at $40 \mathrm{mg} / \mathrm{l}$ it is feasible to state CI AZOL 5010V and Sonkor 9011 comparability.

Table 2. Comparison results of independent CI testing using bubble test with hydrocarbon phase presence.

\begin{tabular}{cccccc}
\hline \multirow{2}{*}{ CI description } & CI Manufacturer & \multicolumn{2}{c}{ IPTER } & \multicolumn{2}{c}{ CAPSIS } \\
\cline { 3 - 5 } & & \multicolumn{2}{c}{ CI protection effect, \%, 20 and 40 mg/l } \\
\cline { 3 - 5 } & Nalco & 0 & 40 & 28 & 47 \\
\hline Corexit SXT 1003 & Opytnyi zavod neftekhim & 96 & 98 & 88 & 99 \\
Sonkor 9011 & Klariant & 87 & 98 & 87 & 94 \\
Dodicor 4712 & Kotlas chemicals plant & 86 & 95 & 83 & 97 \\
AZOL 5010V & & & & & $\mathbf{4 0}$ \\
\hline
\end{tabular}

The CI protection effect measurement results obtained during the bubble test for the combined corrosion mechanism testing with hydrogen sulfide and carbon dioxide present are shown in Table 3.

The test reservoir water from a field developed by Buzulukneft oil and gas production company, Orenburg region, was used as a test solution for the mixed corrosion tests:

\begin{tabular}{ccccc}
\hline Component & $\mathbf{N a C l}$ & $\mathbf{C a C l}_{\mathbf{2}}$ & $\mathbf{M g C l}_{\mathbf{2}}$ & $\mathbf{N a H C O}_{\mathbf{3}}$ \\
\hline Quantity, g/1 & 21.01 & 1.02 & 0.31 & 0.04 \\
\hline
\end{tabular}

$\mathrm{H}_{2} \mathrm{~S}$ concentration of $200 \mathrm{mg} / \mathrm{l}$ was used during a test described in paper [5]. The tests were conducted with $\mathrm{CO}_{2}$ partial pressure of $0.1 \mathrm{~atm}$. Initial corrosion rate: $0.38 \mathrm{~mm} /$ year. Hydrogen sulfide presence was achieved by $1 \%$ of $\mathrm{H}_{2} \mathrm{~S}$ contained in gas continuously fed to the cell. Initial corrosion rate of $0.5-0.7 \mathrm{~mm} /$ year was observed during the experiments described in the paper [6]. 
Based on the results shown in Table 3, the CI efficiency comparison at $40 \mathrm{mg} / \mathrm{l}$ leads to absolutely opposite conclusions:

- IPTER - more benefits of Sonkor 9011 and Cortron-KRN-214W CI;

- CAPSIS - more benefits Corexit SXT 1003 and Dodicor 4712.

Table 3. Comparison results of independent CI testing using bubble test in presence of hydrogen sulfide.

\begin{tabular}{cccccc}
\hline \multirow{2}{*}{ CI description } & CI Manufacturer & \multicolumn{2}{c}{ IPTER } & \multicolumn{2}{c}{ CAPSIS } \\
\cline { 3 - 5 } & & \multicolumn{2}{c}{ CI protection effect, \%, 20, 40 and 80 mg/l } \\
\cline { 3 - 5 } & Nalco & 79 & 82 & 98 & 98 \\
\hline Corexit SXT 1003 & Opytnyi zavod neftekhim & 82 & 88 & 95 & 97 \\
Sonkor 9011 & Klariant & 82 & 82 & 97 & 98 \\
Dodicor 4712 & Champion technologies & 73 & 85 & 94 & 98 \\
\hline
\end{tabular}

\section{Conclusions}

CI development and field application issues should not be limited to ensuring highefficiency protection effects of CIs which is necessary but is not the only condition for efficient field applications.

This paper demonstrates that in addition to the properties measured at the lab, pilots and field corrosion monitoring are vital for understanding process parameters of CIs and protected pipelines.

In order to properly manage the arrange inhibitor protection the following additional, rarely or insufficiently used information is required:

$>\zeta$-distribution coefficient between the water and hydrocarbon phases;

$>\rho-$ density of the protection film on pipeline inner surfaces that provides necessary protection;

$>$ hydraulics and process parameters of protected pipeline operation, including the flow rate, $\%$ of water content, emulsion content and solids concentration;

$\delta$ - dimensionless factor $-\mathrm{CI}$ consumption per unit of weight of the solids;

$>$ Emulsion properties: the average size of oil and water drops and their quantity per unit volume;

$\rho_{\mathrm{o}-\mathrm{w}}-\mathrm{CI}$ density at the oil/water interphase boundary, $\mathrm{g} / \mathrm{m}^{2}$,

duration of the protection film formation on pipeline surfaces;

$\rightarrow$ CI residual content in the water phase at test points of protected pipelines. 
The following initial data is also acquired to perform lab testing:

CI residual content in the water phase after tests completion;

$\rho_{\mathrm{ms}}$ and $\rho_{\mathrm{a}}-$ density of the film on samples and cell walls and equipment, $\mathrm{mg} / \mathrm{cm}^{2}$.

Currently, reliable and affordable measurement techniques are available for only a few of the indicators listed above. Nonetheless, these indicators can be evaluated with lower precision or calculated using available initial data.

Potentials of higher efficiency inhibitor protection achieved by using more sound and specific conditions-oriented CIs, selection of more efficient CIs for specific environments of protected pipelines and proactive CI field application business processes exceed additional costs associated with computation, measurement and engineering required for the CI material balance evaluation described in this article.

\section{References}

1. I. S. Sivokon and N. N. Andreev, Int. J. Corros. Scale Inhib., 2012, no. 1, 65. doi: 10.17675/2305-6894-2012-1-1-065-079

2. I. S. Sivokon and A. Pitersen, Corrosion inhibitors. Pipes will last longer, "Novator" journal on TNK-BP technologies, 2005, no. 4, 14, Moscow ((in Russian).

3. $R \& D$ report "Determination of the efficiency of inhibitor protection on pipelines operated for a long time”, GUP "IPTER”, Ufa, 2012 (in Russian).

4. Report "Results of quality acceptance test of corrosion inhibitors", Nizhnevartovsk, 2011, NizhnevartovskNIPIneft (in Russian).

5. $R \& D$ information report "Analysis of efficiency of corrosion inhibitors used at TNK-BP oil fields”, Stage 5, Laboratory tests, GUP IPTER, Ufa, 2005 (in Russian).

6. Independent corrosion inhibitor evaluation, CAPCIS, Ref. JP4463, December 2005. 